\title{
A Design of Wireless Sensor Network for the Internet of Things
}

\author{
Lei Lu, Qing Zhou, Xingzhen Tao \\ Jiang Xi College of Applied Technology, Ganzhou, China
}

Keywords: Internet of Things; wireless sensor network; operation design

\begin{abstract}
The design of wireless sensor network is growing more mature over time accumulating and technology developing while still is threatened by the future demand. In this paper, the author discussed the wireless sensor network design for the Internet of Things by combining the Internet of Things with the wireless sensor networks. In this way, the wireless sensor network can provide a better service of collecting information and connecting communications.
\end{abstract}

\section{Introduction}

WSN (Wireless Sensor Network) is constituted by vast immobile and moving sensors self-organizing and multi-hopping which are the most important technical form in the underlying network of Internet of Things. The growth of WSN technology enables itself to get the necessary information under any occasional time, location and circumstance and performs a guiding role for the Internet of Things future development.

\subsection{IOT (Internet of Things)}

As an important part of new-age information technology, key stage of current trending and crucial component of technology development, IOT is a network of connecting things together and realizing online resources sharing, which explains it as a extend of traditional Internet. Communication technologies of IOT like information collecting and recognizing are widely used by the public and has generated a progressing information time. It keeps developing itself, exploring new outlets and creating new opportunities for researchers in this field.

\subsection{WSN (Wireless Sensor Network)}

The work mechanism of WSN can be easily explained as through the end to perform the sensing and inspection function, which means the network design is set up as sensitive. With more improvement of the network by connecting wireless network and the wired network together, the network system will be more guarded and clearer. In the meanwhile, the WSN can be used to data collecting, processing and transmission of low cost and high applicability. Furthermore, it will inspect the data in the collecting and transmission process that further protects the stability and safety of the network. As for now, China is very strict with wireless sensor networks, and requires its performance and technology to be strong enough to meet the needs of our country's development and progress.

IOT allows people to easily obtain and share information everytime and everywhere and WSN is responsible for the network safety. If the WSN can be realized on IOT, circumstances will be improved better in the safety and convenience aspects, which improves IOT technology in return. The following Fig 1 show the practical functions of WSN in the underlying network of IOT through targets, messages and encapsulation three concepts. 


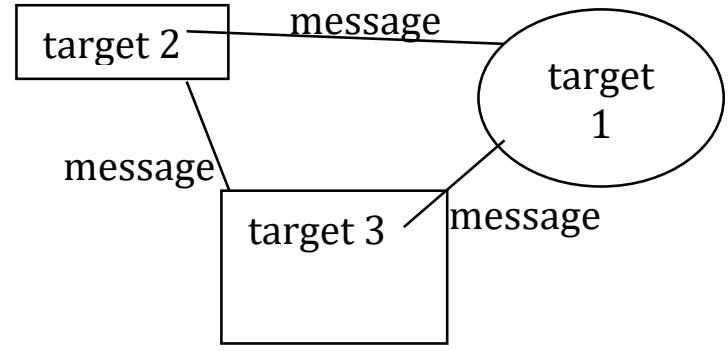

a. interact operation among targets

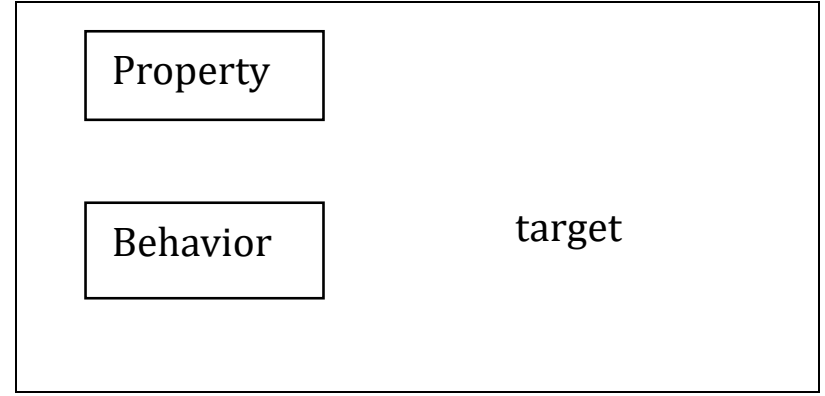

b. encapsulation of target

Fig 1

\section{Key Techniques}

Key techniques in WSNs mainly are constituted from three aspects. Here is the whole content in the following table 1 .

Table 1

\begin{tabular}{|l|l|l|}
\hline $\begin{array}{l}\text { information collecting system } \\
\text { design }\end{array}$ & $\begin{array}{l}\text { network communication protocol } \\
\text { design }\end{array}$ & network service support \\
\hline network model design of WSNs & network layer design & time synchronization mechanism \\
\hline platform and operation system & data link layer design & network node localization \\
\hline standardization of node design & transport layer design & network topology coverage \\
\hline data storage & physical layer design & data fusion and compression \\
\hline test bed design & cross layer optimization design & network security mechanism \\
\hline diagnostics and debugging support & & \\
\hline
\end{tabular}

Then the author will explain several typical techniques.

\subsection{Network communication protocol}

In the process of network information technology, network communication system will be a chaos without rules at all if there's no defined network protocol constraints, which will greatly hinder the development and process of network information technology. Wireless sensor network system is generally based on the TCP and IP protocol as a basic network protocol which is conducive to the transmission of network information. In the transmission process, each node of the wireless sensor network can be fully utilized on the fundamental routing protocol, so as to improve the efficiency of the transport network. The Internet of things acts as an important tool of information processing and wireless sensor network is the core target in this research. In the process of data processing, the common regulation and monitoring of network protocol and routing protocol are required in order to make a more diversified needs are met and to make the information more quickly and effectively in accordance with the public. Therefore, network communication protocols are essential in the design of WSN.

\subsection{Network safety design}

Network security is an important issue in the process network information technology development that absolutely can not be ignored by everyone. People should aware that the openness of the network will threaten the safety of personal information while they are enjoying the benefits brought by the information sharing technology, which is of great potential safety problems and will directly harm the interests of users even cause great losses. Therefore, for this problem, the wireless sensor network calls for a great need for encryption technology to protect the security of network information and prevent information steal by enhancing the network security. With encryption and message authentication in network security design, we can ensure the safety and integrity of 
information to a certain extent which contributes itself to be a must in the WSN for IOT.

\subsection{Networking positioning technique}

In the process of daily using, the public will have a certain psychological dependence on wireless sensor networks. The use of wireless sensor network in our life is very convenient under many circumstances. The introduction of this positioning technology mainly works to collect the node data of wireless sensor network and accurately points out the occurrence location forming itself as the core technology. This technology refers to the modern computer techniques and shows a very accurate analysis of location information. People can even use this technology in real-time location monitoring so as to better conduct a data collection and collation work. The introduction of this technology can greatly promote China's scientific research work, continuously provide real-time reliable data for China's science business. So network positioning technology is rather important for the WSN design for IOT.

\subsection{Network topology technique}

The so-called network topology is defined as a hierarchical topology structure by employing the technology of network transmission equipment connecting to the physical layout. Due to the certain order in the point connection in the network structure, the application of network topology in WSN can eliminate redundant parts in the network to some extent. And also can make the network communication channels be expanded to greatly improving the work efficiency of network information transmission and wireless sensors. With the improving WSN technology, network topology technology is constantly optimized attracting lots of attention from the public. A wireless sensor with a well-performing network topology technology costs less node energy. The routing efficiency is higher and the network performance is much better. So the introduction of network topology technology is a key step for wireless sensor networks to meet the development needs of the information age.

\section{Optimization Design}

IOT technology shows a strong popularity with the public alongside the rapid development of modern network technology and artificial intelligence. WSN keeps continuous optimization and upgrading basing on this fact as a fundamental for IOT. This part has conducted a explicit and full discussion on the design from the following three aspects: information collecting system design, network service support optimization and network communication protocol design.

\subsection{Network communication protocol design}

A reliable and efficient network protocol has a crucial role for the application of wireless sensor network. Each node in a sensor network can transform the message to the sink node through multi hop in communication protocol stack. So the protocol design of wireless sensor network should be energy efficient. At the same time, focus on communication the design of wireless sensor network protocol stack in China should be put on the data link layer, transport layer and network layer as well as those interactions between the cross layers. The data link layer can be constructed to form the bottom of the junction structure, which can be realized through its access limitation. At the same time, it can also control the node to keep the work mode always at the best. The transport layer can greatly ensure the safety and reliability of data transmission and the network layer can make the transmission path in a reasonable and safe direction. In general, if the use of IOT technology can help to establish a network communication protocol satisfying various needs, it can effectively reduce the complexity of the design of wireless sensor network and improve the work efficiency of wireless sensors. In addition, the communication protocol and IPV6 can also be further optimized by using IP technology, in this way to make the information and data being collected more reliable and valuable on one side, the flexibility of data transmission being not destroyed. 


\subsection{Information collecting system design}

For one node in WSNs, some of the more important system design must use a variety of crucial technologies like reasonable design of wireless sensor network model and platform, which is the only way to better carry out relevant information and data acquisition and processing, so that the entire wireless sensor network is more practical.

\subsubsection{Model design for WSN}

In order to grasp the characteristics of network application, it is necessary to establish a model of WSN. It can also make the network structure forming a good pattern, which can be applied to a variety of network environment and greatly improve the accuracy through continuous improvement. The design of wireless sensor network model can be classified according to its different communication functions to better establish a service-centered model and to provide a better experience for users. The most common network modeling is the ones based on cluster tree structure whose framework is relatively flexible with layered nodes consuming more energy. Under these conditions, these nodes must be set as equal to extend the network lifetime. This can not only make the energy distribution even, but the strong convergence of dynamic network topology condition exists still. This kind of model is the right one for both WSN design and actual users.

\subsection{2 sensor system design}

Every sensor node of each manufacturer can not be created as the same in the communication module, the microprocessor and storage space. A perfect fusion of a variety of advantages in the sensor is not easy. One node is different from other in the hardware. These differences make the realization more difficult. But if the wireless operating system the sensor network can provide the necessary support to the sensor platform, it can make sense data can be handled effectively, which would solve the problem with constrained node data. At present, the storage mode of wireless sensor network design includes: the network external storage, local storage and data centric storage. Overall, the third storage method is more reasonable. Therefore in order to keep a low consumption of communication efficiency and energy, it can be done to set a limitation on sensor node resource and searching need. Finally, we can design and test the test bed for researchers to do relevant experiments, so that we can better verify the effectiveness and feasibility of various algorithms and network applications.

\subsection{Network service support optimization}

In the WSN design, the sensor node configuration, processing and other services can be used to monitor and coordinate the nodes in energy and resource utilization, providing a guarantee for the efficient use of the Internet. Data control service in the design of wireless sensor network also occupies a very important position. So it can be said that the network service support for the maintenance of network system has a very important role, which is necessary to gradually achieve the optimization problem. The functional performance of the WSN design turns out to be very reliable, especially in the data transmission, control, treatment and other aspects. Therefore it is necessary to optimize the network service for the realization of data protection. Another problem is the coverage issue. Wireless sensor network coverage directly affects the number, position, energy and connectivity of sensor nodes, so we must ensure the monitoring region to achieve full coverage in the high security situation. By optimizing the network service can greatly improve the security of wireless sensor networks and efficiency and create better user experience.

\section{Conclusion}

Given the background of information technology forming a strong popularity in current society, WSN is increasingly welcomed into people's daily life and regarded as a strong power in social development as the basic technology in IOT. Enlightened by this fact, the optimization and upgrade of WSN for IOT can both benefit the network technology progress and actual daily life. 


\section{References}

[1] Zhihong Qian, Yijun Wang. A Review of Wireless Network Sensors for Internet of Things [J]. Journal of Electronics and Information Technology. 2013, 17 (01): 215-227.

[2] Hailan Yang. A Analysis on WSNs Safety and Reliability of IOT [J]. Logistics technology. 2015, 21(03):294-296.

[3] Wei Xiong. A Review of WSN for IOT [J]. Electronic Technology \& Software Engineer. 2015(14):27-27.

[4] Yijun Wang. A Research on Time Synchronization and Addressing Strategy of WSN for IOT [J]. University of Jilin. 2012. 